\title{
The Effect of Vibro-Finishing Treatment on The Porosity of the Surface Layer of Powder Metallurgy Products
}

\author{
Yuriy Ankudimov ${ }^{1 *}$, Irina Sadovaya ${ }^{1}$, Dieudonne Essola ${ }^{2}$ and Tatyana Bagdasaryan ${ }^{1}$ \\ ${ }^{1}$ Don State Technical University, 344000, Rostov-on-Don, 1 Gagarina prospect, Russian Federation \\ ${ }^{2}$ University of Douala, Douala, Cameroon
}

\begin{abstract}
The article is devoted to the methods of surface plastic deformation treatment to change the porosity of parts obtained from powder materials. Functional dependences between the concentration of the solid part of the system and the size of the plastic print on the example of vibro-finishing and hardening treatment (VF and HT) are established. The reduction in porosity upon hardening of the surface material is experimentally confirmed. The results can be used to predict the surface porosity of powder metallurgy products that have undergone additional processing of SPD, as well as for further studies of the field of application of (VF and HT). Based on the particle interaction in the working medium with the part surface made from powder material, we obtained functional dependence of the density coefficient of the surface layer of the powder material after (VF and HT). Experimental studies were carried out on samples of powdered iron PK6. The porosity of the samples was determined by stereoscopic metallography, microhardness of the surface layer - on hardness tester. Everything was performed by standard methods. The results were presented in a series of porosity charts. Maximum hardening is $30 \%$ of almost twofold porosity reduction. The experts in the field of powder metallurgy may be interested in the obtained results.
\end{abstract}

\section{Introduction}

Structural sintered parts made of iron-containing materials make up the main part of powder metallurgy products. These parts are significant for instrument making industry and for some spheres of metal working industry also. However, the production of powder products is still limited, although there is a tendency of its constant increase due to the improvement of the quality of powder products. The properties of sintered parts are determined primarily by porosity and composition. In addition, these properties can be widely changed by varying the manufacturing technology (Tab.1).

Table 1. Methods of production, porosity and application of some powder materials [1]

\begin{tabular}{|c|c|c|c|}
\hline $\begin{array}{c}\text { Production } \\
\text { methods }\end{array}$ & Material & $\theta, \%$ & Application \\
\hline
\end{tabular}

*Corresponding author: Ankudimov1950@mail.ru 


\begin{tabular}{|c|c|c|c|}
\hline $\begin{array}{l}\text { Filling of } \\
\text { power or } \\
\text { fibers }\end{array}$ & $\begin{array}{l}\mathrm{Cu}, \mathrm{Ni}, \mathrm{Fe} \\
\text { and their alloy }\end{array}$ & $\leq 60$ & Filters \\
\hline Pressing & $\begin{array}{l}\text { Bronze, iron } \\
\mathrm{Fe}, \mathrm{Fe}+\mathrm{Cu}, \\
\text { copper alloy }\end{array}$ & $\begin{array}{l}\leq 30 \\
\leq 20\end{array}$ & $\begin{array}{c}\text { Self-lubricating bearings } \\
\text { Small strength items } \\
\text { (mainly for static loads), friction and } \\
\text { antifriction materials. }\end{array}$ \\
\hline $\begin{array}{l}\text { Pressing }+ \\
\text { repressing }\end{array}$ & $\begin{array}{c}\mathrm{Fe}+\mathrm{Cu}, \mathrm{Fe}+\mathrm{Sn} \text {, alloys } \\
\mathrm{Fe}+\mathrm{Cu}+\mathrm{C}, \\
\mathrm{Fe}+\mathrm{Cu}+\mathrm{Ni} \\
\mathrm{Fe}+\mathrm{Ni}+\mathrm{Mo}+\mathrm{C}\end{array}$ & $\leq 5$ & $\begin{array}{l}\text { Medium strength and high durability items. } \\
\text { Items of high durability, cemented items }\end{array}$ \\
\hline $\begin{array}{l}\text { Pressing + } \\
\text { infiltration }\end{array}$ & $\begin{array}{l}\mathrm{Fe}+\mathrm{Cu} \text { (impregnation } \\
\text { with copper alloys) }\end{array}$ & $\leq 5$ & $\begin{array}{l}\text { Items of high durability, dispersion } \\
\text { hardening }\end{array}$ \\
\hline Hot forging & $\begin{array}{c}\text { Steel Ni - Mo, } \\
\text { Steel Ni - Mo - Mn }\end{array}$ & $\leq 1$ & High strength items for dynamic load. \\
\hline
\end{tabular}

The production of powder products can reduce or completely eliminate waste for cutting processing. The production of simple (in shape) parts saves at least $15 \%$. The production of complex parts, when the traditional manufacture requires different cutting methods, saves up to $50 \%$.

Additional processing allows to expand application of powder products and to improve their properties. It is important for all infeasible technique of pressing contour parts that are performed with removal of chips. In addition, wear-resistant, anti-corrosion and decorative coatings can be applied to sintered parts with porosity $<10 \%$. Powder iron and steel products can be hardened directly or after cementation. With the appropriate materials, precipitation hardening can be carried out, especially for sintered materials, infiltrated in metal.

\section{Materials and methods}

To obtain a more hard-textured part and an accurate size, one can apply a double cycle of pressing and sintering. Mechanical properties can be improved in two ways - by reducing porosity and doping.

Additional processing includes a number of operations. In particular, with high requirements for dimensional accuracy, it is advisable to remove the burrs from the shaped parts by vibration abrasive grinding. Removing chips from low-alloy and thermally untreated parts with a metal blade tool does not cause difficulties. Steel powder doped with sulphur is also good here.

The heat treatment is of particular importance, it improves the mechanical properties, wear resistance and corrosion resistance of powder materials. One can harden sintered steel as well as cast. More often, however, surface hardening is used, which increases wear resistance. There are other thermochemical methods except for surface hardening of iron powder, such as: nitrogenization, ni-carbing and boronizing, leading, as in the same processing cast steel, to a part size change. It should be taken into account in producing parts with accurate dimensions. In the same way, water vapor oxidation acts, it is used to improve wear resistance and corrosion resistance. It is also possible to use the oxidation method as a pre-treatment before galvanic coating. The oxide-magnetite film fills the pores well bonded to the metal and protects it from corrosion like varnishes, oil and fat. Due to semiconductor property of this film, copper, nickel or chromium can be electroplated.

An effective way to reduce the porosity of products from powder materials is surface plastic deformation (SPD), by various technological methods $[2,3,4]$. The experimental 
study of possibility of using one of the methods of (SPD) - vibratory finishingstrengthening processing technology of the manufacture of parts powder metallurgy with the aim of reducing surface porosity and compaction of the surface was carried out on samples of powder iron PK6 porosity of $22-25 \%$, a hardness of 70-80 HB.

\section{Research result}

The porosity change in the depth of deformation $h$ of the part surface layer in implementing working medium particle can be represented in the following form (Fig.1).

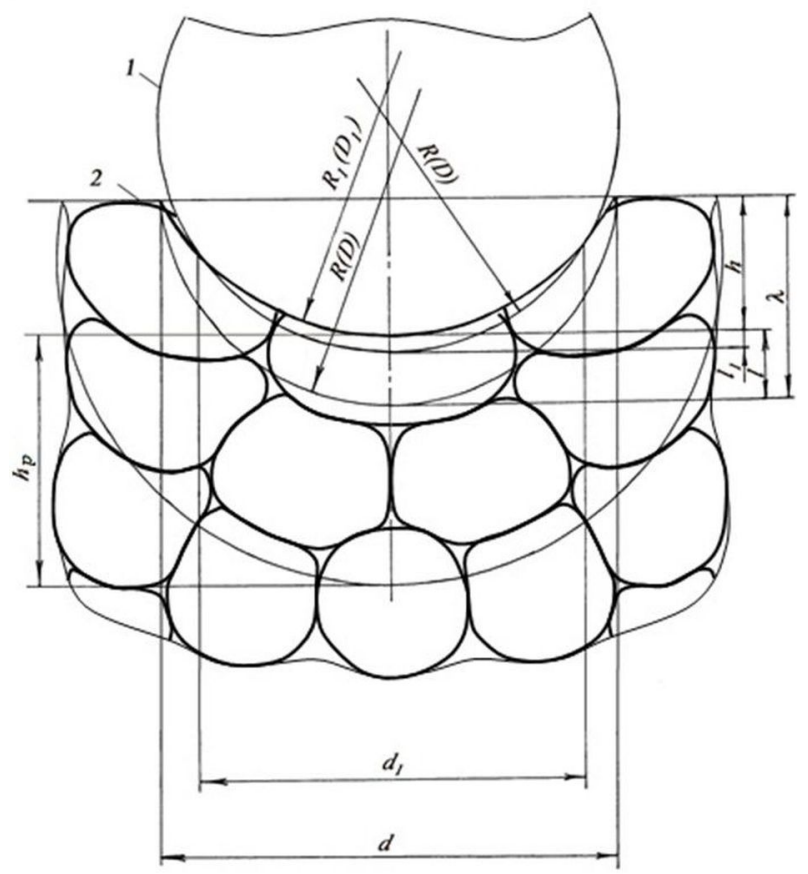

Fig. 1. Scheme of interaction of the working medium (1) with the surface of the porous part (2): $R(D)$ is the radius (diameter) of the particles of the working environment, $R 1(D 1)$ - radius of curvature of the restored print, $h$ - depth of the restored print, $h_{p}$ - depth of plastic deformation zone, $l$ - elastic component of the imprint depth, $\lambda$ - the penetration depth of the particle of the working medium, $d-$ diameter of the recovered print, $d_{l}-$ the diameter of the unrestored print.

In the initial state, the structure of the material is presented in the form of particles close to the spherical shape, interconnected by "bridges" of interparticle contact formed as a result of sintering of the material. The relative density of such material will be characterized by the ratio of the volume of the solid part of the system of sintered particles to the total volume of the system:

$$
v=\frac{V_{s . p .}}{V_{o}} .
$$

The porosity of such a system is expressed as

$$
\theta=(1-v)
$$


and will be within $0 \leq \theta \leq 1$.

When the particles of the processing medium interact with the surface during vibration finishing and hardening treatment (VFHT), traces in the form of holes (prints) are formed, covering the entire surface, they create a stress-strain state in thickness.

When the surface is completely covered with prints, it can be assumed that the compression of all layers in thickness on average will correspond to the deformation of the axial compression under one print.

For dynamic SPD methods, the dependence proposed by I. V. Kudryavtsev is known, which connects the zone of plastic deformation with the size of the plastic imprint $h_{p}=k_{p} \cdot d$ (for iron and its alloys $k_{p} \approx 1.5$ ).

For vibrating finishing and hardening treatment of parts made of powder materials, it gives conservative value of the depth of plastic deformation. Our studies show that the depth of plastic deformation at (VFHT) captures only a few layers of the particles of the near-surface layer. Without much damage to the accuracy, the depth of plastic deformation zone can be adopted:

$$
h_{p} \approx k_{p} \cdot a_{s}
$$

where $k_{p}$ - the coefficient is $1 \ldots 3, a_{s}$ - average particle size of the initial powder of sintered material.

It is obvious that the deformation of the powder particles and pore filling will decrease as the distance from the surface to the depth of the material, and the volume of the solid part of the $V_{s . p .}$. system does not change, but only leaves the volume of the recovered imprint.

Based on the full coverage of the prints with a diameter $d$ of the entire surface, the density of the initial powder material in the volume of the plastic deformation zone is expressed by the ratio:

$$
v_{o}=\frac{V_{s . p .}}{V_{0}} \approx \frac{V_{s . p .}}{\pi \cdot \frac{d^{2}}{4} \cdot\left(h+h_{p}\right)},
$$

where $h-$ is the depth of the restored print.

The density of the surface layer of the powder material after (VFHT):

$$
v=\frac{V_{s . p .}}{V}=\frac{V_{s . p .}}{\pi \cdot \frac{d^{2}}{4} \cdot h_{p}} .
$$

The change in the porosity of the surface layer, based on the linearly decreasing concentration of the solid part of the system, squeezed out of the volume of the restored hole in depth, taking into account (2) will be:

$$
\Delta \theta=\theta-\theta_{0}=\frac{2 h}{h_{p}} \cdot(\theta-1) \cdot\left(1-\frac{x}{h_{p}}\right),
$$

where $x$ - is the distance of the deformed layer from the surface.

The size of the plastic imprint (its diameter, depth) depends on the power and energy parameters of the method of surface plastic deformation - the force of indentation when 
rolling the ball, the amplitude-frequency modes during vibration hardening, etc. If we know the nature of these dependencies, we can predict the degree of porosity reduction during processing by one or another method of (SPD).

The process of VFHT [2,5] consists in the sequential application of a large number of micro-shocks on the surface of the machined parts by a set of solid particles (steel balls, fractions, etc.) of the working medium, they perform low-frequency oscillations with an amplitude of 2-15 $\mathrm{mm}$ and a frequency of $15-50 \mathrm{~Hz}$.

The following data on the control of porosity of samples by stereoscopic metallography (Fig. 2) and microhardness of the surface layer (Fig. 3).

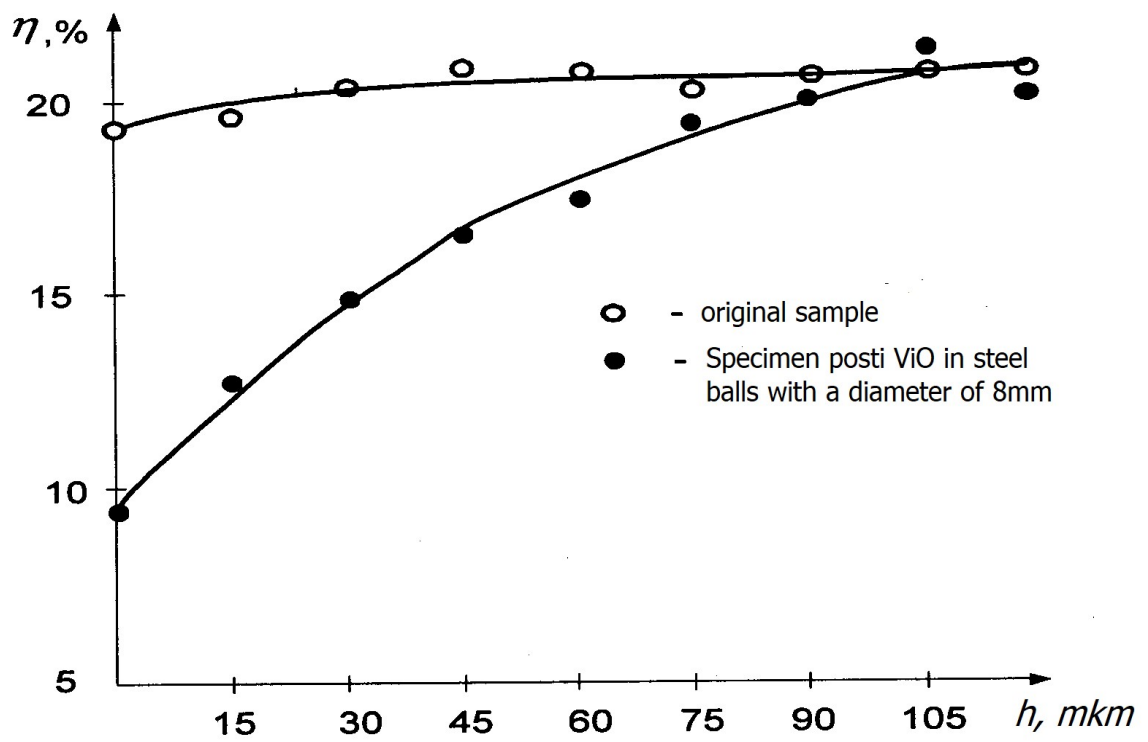

Fig. 2. Dependence of the porosity change of the samples on the depth of the surface layer 


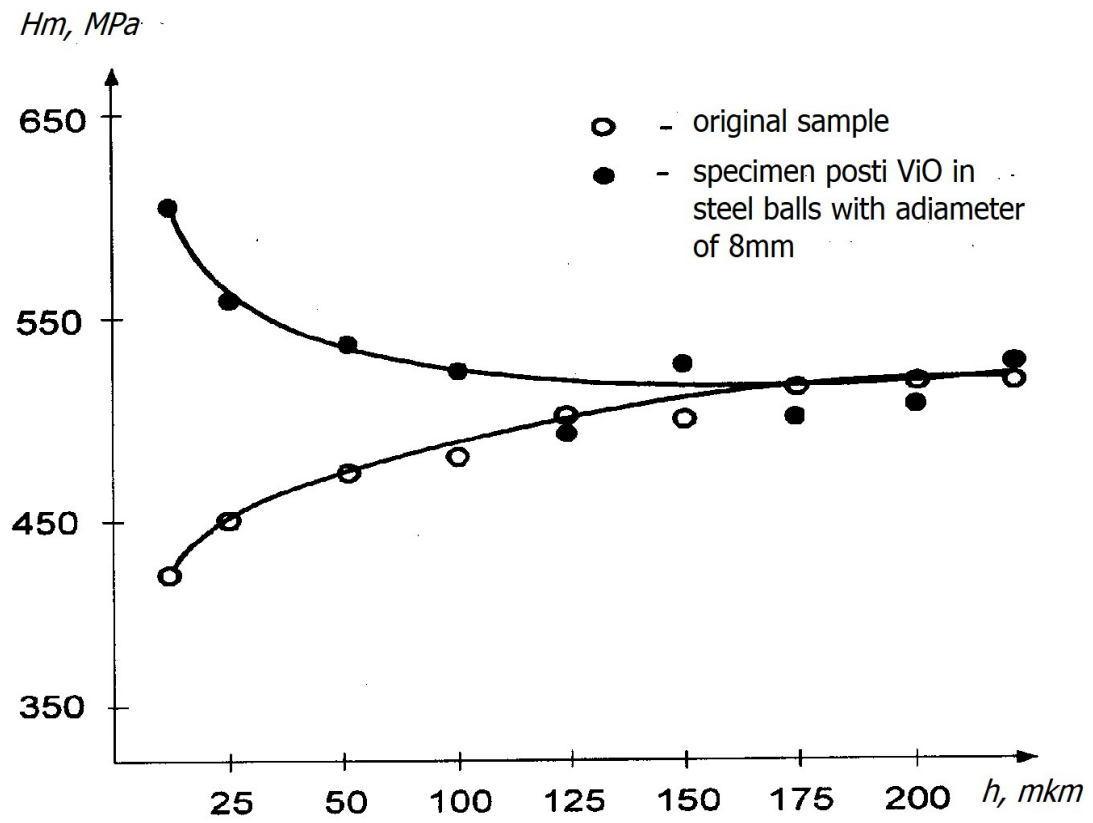

Fig. 3. Dependence of microhardness change of the surface layer.

\section{Discussion and conclusion}

Based on the particle interaction in the working medium with the part surface made from powder material, we obtained functional dependence of the density coefficient of the surface layer of the powder material after (VFHT).

As the plastic deformation spreads along the depth of the imprint, the spherical shape of the sintered particles of the powder material is textured, as a result, the ratio of the volume of the solid part of the sintered particles system to the total volume of the system increases. The obtained dependences indicate that the plastic deformation zone extends to a depth up to 100 microns with the studied process parameters. Detailed analyses revealed the porosity decrease of the material and increase its microhardness at (VFHT). Maximum hardening is $30 \%$ of almost twofold porosity reduction.

The theoretical and experimental studies have shown the technical feasibility and practical feasibility of using VFHT to seal the surface of parts made of powder materials. The obtained results can be used to predict the surface porosity of powder metallurgy products that have undergone additional processing of SPD, as well as for further studies of the field of application of VFHT.

\section{References}

1. V.S. Panov, Technology and properties of sintered materials (MISIS, M, 2001)

2. A.P. Babichev, Yu. P. Ankudimov, I.V. Sadovaya, Bul. RGATU, 2, 52 (2017)

3. K. Hamouda, T. Sayah, J.P.Ankudimov, P.J. Ankudimov, A.P. Babichev Defect and Diffusion Forum, 297, 1103 (2010)

4. V.A. Lebedev Technologyof Dynamic Methods of Surface Plastic Deformation (DSTU, Rostov-on-Don, 2006) 
5. A.P. Babichev, E.A. Babichev, Basic Concepts of Vibration Technology (DSTU, Rostov-on-Don, 1999)

6. Yu.P. Ankudimov, Yu.G.Chernega, Integrated vibration wave technologies in mechanical engineering, metalworking (DSTU, Rostov-on-Don, 2015)

7. V.N. Bagaev, Yu.A.Buevich, V.V. Tetyukin, Mag. Hydr. 2, 35 (1986)

8. V.A. Lebedev, Yu. P. Ankudimov, Engineering and technosphere of the XXI century (DNTU, Donetsk, 2012)

9. J.M. Vernigorov, B.G. Gasanov, S.S. Baev, Sol. S. Ph. 265, 1020 (2017)

10. V. V Ivanov., N.S. Dontsov, A.V. Kirichek Key Eng. Mat., 736, 105 (2017)

11. V A Lebedev, V V Ivanov, V P Fedorov IOP Conf. Ser.: Mat. Sc. Eng. (2016)

12. M. Tamarkin M, E.Tichshenko,V.Fedorov IOP Conf. Ser.:Mat. Sc. Eng. (2015)

13. A.A.Dmitrovich A.A., A.L.Terekhov, L.M. Shmagin Pow. Met., 7, 61 (1983)

14. A.V. Stepanenko, E.B.Lozhechnikov, E.V. Lozhechnikov Pow. Met., 11, 97 (1984)

15. V.A.Genkin, V.N. Gurlena, A.A. Pow. Met., 6, 58 (1982) 